\title{
Investigación-acción cooperativa: aprendiendo de una experiencia fallida*
}

\author{
Marco Antonio Villalta Paucar \\ Universidad de Santiago de Chile, \\ Santiago, Chile \\ Marisol Ortega Bahamonde \\ Escuela Tranapuente, \\ Carahue, Chile
}

\begin{abstract}
Recibido: 11 de enero del 2019 / Aceptado: 11 de marzo del 2019 doi: 10.26439/persona2019.n022(1).4088
\end{abstract}

El presente articulo tiene por objetivo identificar aprendizajes de uso de la investigación-acción colaborativa (I-A-C) entre una escuela rural y una universidad estatal, desde el análisis de fortalezas y desafíos de la experiencia. Metodológicamente, se trata de un estudio de caso de investigación-acción. Se relata analíticamente la experiencia, en la que se reconocen fortalezas: el reconocimiento recíproco, el aprecio del otro institucional como colaborador de un proyecto con metas compartidas, el compromiso de los actores educativos interinstitucionales para con los estudiantes. Asimismo, desafíos: la gestión de contingencias internas de cada institución en la coordinación interinstitucional y las diferencias institucionales para distinguir problemas y metas de intervención. Desde la experiencia, se distinguen los siguientes aprendizajes: clarificar las metas de la colaboración con criterio de realidad por sobre el justo deseo de las grandes transformaciones, conocer los protocolos y tiempos administrativos institucionales que inciden en la colaboración interinstitucional. investigación-acción / cooperación / universidad / escuela / educación

\footnotetext{
* Estudio realizado en el marco del Concurso de Investigación e Innovación Asociativa Universidad-Escuela, de la Universidad de Santiago de Chile.
}

Correo electrónico: marco.villalta@usach.cl 


\section{Collaborative Action Research: Learning from a Failed Experience}

The aim of this article is to identify learning situations used in collaborative action research (CAR) in a rural school and a state university, based on the analysis of the strengths and challenges of experience. From a methodological point of view, this is a case study of action research. The experience is analytically assessed by recognizing the strengths - reciprocal recognition, appreciation of the other institutional body as a project partner with shared goals, and commitment of the inter-institutional educational actors to the students- and the challenges -management of each institution's internal contingencies for inter-institutional coordination, and institutional differences to distinguish intervention problems and goals. Based on the experience, the following learning situations are recognized: clarifying the goals of collaboration with reality criteria over the fair desire to achieve great transformations, and knowing the institutional administrative times and protocols that affect the inter-institutional collaboration. 


\section{INTRODUCCIÓN}

La investigación-acción es una respuesta para innovar en los procesos educativos con sentido de pertinencia cultural, y emerge como una perspectiva transformadora de la realidad, social y educativa (Labrador-Piquer y Andreu-Andrés, 2014; Martínez, 2014).

La investigación-acción ha tenido en la educación diferentes enfoques, tales como la investigación-acción del docente y la investigación-acción cooperativa, especialmente entre escuela, familia y comunidad para promover una educación inclusiva (Francke y Ojeda, 2013; Simón, Giné y Echeita, 2017). Esto es así debido al carácter práctico que caracteriza a esta perspectiva de investigación, la cual está en sintonía con las situaciones contingentes de la vida cotidiana en las escuelas.

En la investigación-acción de tipo cooperativo (I-A-C), la escuela y la unidad de investigación (la universidad) establecen alianzas de trabajo en las que se coordinan la teoría y la práctica, y confluyen para resolver un problema percibido como práctico en la escuela (Gómez, Flores y Jiménez, 1996; Serrano, 1994).

No obstante el carácter contextual de la I-A-C, los requerimientos de su operatividad no se delimitan al problema sentido por los prácticos, sino también a las condiciones de espacio y tiempo para la reflexión de la acción, lo cual cobra particularidades cuando dicha condición depende de la coordinación de dos organizaciones diferentes: la escuela y la universidad. Ambas tienen contingencias, prioridades, ritmos administrativos propios y dependientes de unidades superiores y centralizadas de administración.

El presente artículo tiene por objetivo identificar aprendizajes de uso de la investigación-acción colaborativa (I-A-C) entre una escuela rural y una universidad estatal, desde el análisis de fortalezas y desafíos de la experiencia.

\section{Investigación-acción cooperativa: algunos elementos de referencia}

El origen de la investigación-acción se atribuye al trabajo de Lewin, a comienzos del siglo $\mathrm{xx}$, el cual se define como una alternativa a las metodologías de investigación positivas. Él las describió en cuatro fases: (a) planificar, (b) actuar, (c) observar, (d) reflexionar. De ellas, el foco era la reflexión del práctico sobre sus prácticas (Gómez et al., 1996). La investigación-acción se basa en una concepción de la ciencia como una construcción que produce conocimiento sustentado en la praxis social (Fals, 1999). Se reconocen tipos de investigación-acción que corresponden a diversas corrientes de pensamiento, vinculados a los contextos socio-históricos que los hicieron posibles, así como a tipos de prácticas sociales. Entre ellos, destaca la variedad de propuestas en el campo educacional (Gómez et al., 1996; Serrano, 1994).

Específicamente, en el campo educacional se reconocen dos modalidades de investigación-acción: la investigación-acción del profesor, orientada a abordar los problemas susceptibles de cambio en el aula; y la investigación-acción-cooperativa (I-A-C), una forma de investigación participativa que refiere al vínculo entre escuela 
y universidad para abordar un problema de la escuela definido de manera mutua (Fals, 1999; Gómez et al., 1996).

La I-A-C desde la universidad aporta la pertinencia cultural de los conocimientos que esta institución produce al propiciar el diálogo constante entre la teoría y la realidad;, contribuye a la profesionalización de los docentes, defendiendo y dando valor al conocimiento construido en la praxis, y reivindica el papel de los sujetos como constructores de realidad a través de su acción (Fals, 1999; Martínez, 2014). Así, la I-A-C ha sido valorada como respuesta innovadora a las demandas de la política educativa de logro de resultados ajustados a estándares predefinidos (Martínez, 2014).

No obstante, la implementación de la I-A-C entre universidad y escuela es un proceso complejo que Martínez (2014), al sistematizar el estudio de Bálcazar realizado en 2003, resume en seis tipos de tensiones:

1) Son los investigadores quienes deciden la metodología de investigación; 2) el peso de la cultura de investigación positivista colisiona con la idea de involucramiento y acercamiento a los puntos de vista de los actores; 3) muchas veces el pesimismo de los miembros de la comunidad cierra las posibilidades de cambio; 4) la falta de recursos y tiempo para investigar ponen en desventaja a los diferentes participantes; 5) la intervención revela conflictos en la comunidad; y 6) el tiempo para el análisis colectivo de la experiencia no necesariamente es contemplada en los proyectos de investigación. (2014, p. 63)

En tal sentido, la I-A-C es una posibilidad, pero también un desafío para el fortalecimiento de redes de producción de conocimiento que incidan en el desarrollo de prácticas educativas pertinentes y eficaces para la profesionalización de los docentes, el logro de aprendizajes significativos y la valoración de conocimientos basados en la praxis, pero también ajustados a las reglas de la ciencia (Fals, 1999).

\section{Metodología}

Se trata de una investigación-acción en cuyo ciclo ha interesado el análisis descriptivo-interpretativo del proceso de decisiones tomadas y los aprendizajes adquiridos. En consideración con el objetivo del trabajo, los criterios de inclusión estuvieron dados por dos elementos:

- Condiciones institucionales que facilitaran la coordinación entre universidad y escuela. Esto se dio con la convocatoria de la universidad estatal a un concurso abierto para que las escuelas asociadas a ella por diversos proyectos pudieran postular mediante la formulación de un problema inicial que identificaran como relevante de resolver en su escuela y para el cual requirieran el apoyo técnico y financiero de la universidad.

- La convergencia inicial de inquietudes y competencias para abordar problemas referidos al aprendizaje en la escuela. El problema de investigación postulado fue evaluado por un equipo de investigadores de la universidad, en función de la relevancia del problema educativo planteado, los recursos y las competencias para abordar dicho problema inicial expuesto por las escuelas.

Con tales criterios, se seleccionó un conjunto de proyectos de escuela. Este estudio 
se focalizó en la experiencia de una escuela rural, ubicada a aproximadamente a $762 \mathrm{~km}$ de distancia de la universidad estatal. La lejanía geográfica planteaba particulares desafíos al proceso de I-A-C con respecto a la coordinación de las actividades.

Los participantes fueron tres investigadores de la universidad estatal, todos de la región metropolitana de Chile, y tres docentes de la escuela rural de la Región de La Araucanía. Luego de la adjudicación del concurso, el trabajo de coordinación de equipo interinstitucional duró cinco meses, se inició en septiembre del 2016 y concluyó en enero del 2017.

Fuentes de información y procedimiento ético de investigación. Se utilizaron actas de reunión, relatos de experiencia de los docentes participantes, documentos producidos y referidos al proyecto, y un grupo focal con alumnos de segundo y cuarto básico, participantes de una de las actividades realizadas directamente con ellos.

El estudio se ajustó a protocolos éticos de la investigación social. Se concretó con la autorización del director de la escuela rural, el consentimiento de los docentes que intervinieron y de los alumnos participantes menores de edad. Se resguardó la identidad de las instituciones y los participantes en el estudio, por lo que se usaron nombres genéricos para las instituciones, $y$ abreviaturas en las citas con datos textuales de los participantes.

Procedimiento de trabajo de campo. Se organizó en tres fases:

1. Fase de convocatoria y primer contacto. La universidad tomó la iniciativa de convocar un concurso abierto para establecer un vínculo de trabajo con las escuelas. La escuela rural se adjudicó el concurso con un tema orientado al logro en sus alumnos de aprendizajes con pertinencia cultural a través de uso del huerto escolar.

2. Fase de desarrollo. En esta fase se efectuaron las coordinaciones entre el equipo de investigadores de la universidad y el equipo de docentes de la escuela, para concretar la pregunta de investigación, reenfocar los objetivos y revisar las actividades que se realizarían; junto con los procesos de transferencia de recursos que la universidad había comprometido y los recursos de tiempos y capacidad técnica que los docentes y apoyos de la escuela podían implementar.

3. Fase de cierre de la experiencia. Es la fase de sistematización de la experiencia a través de grupos focales, reportes de experiencia, registros fotográficos y actas de reunión que se articularon en un relato que permitió evidenciar los aprendizajes obtenidos de la I-A-C.

Procedimiento de análisis. Se llevó a cabo el análisis de contenido semántico de las fuentes de información. Las técnicas estuvieron al servicio de la reflexión crítica de la experiencia. Se consideraron como unidades de contexto de la información las fases descritas y las actividades específicas que los informantes destacaron en sus reportes o se encontraron en los documentos. Como unidad de registro, se tuvieron en cuenta los discursos referidos a aspectos favorables y problemáticos, así como sugerencias sobre actividades 
específicas, sobre su propia participación, y sobre los resultados del proyecto. Como recurso de apoyo en esta tarea se utilizó el software NVivo 11 Pro.

\section{Resultados}

La presentación de los resultados se organiza en una estructura temporal de fases. Esto permite visualizar cómo fluyen, emergen o se diluyen las unidades de registro definidas a través del análisis de contenido.

Fase de convocatoria e inicio: entre la ilusión y la confusión

En esta primera etapa la escuela rural postuló y se adjudicó el concurso con la propuesta "Aulas interdisciplinarias de aprendizaje en contextos para la valoración de la cultura local y el cuidado del medioambiente". Su objetivo general era generar espacios de aulas interdisciplinarias, utilizando estrategias de articulación pedagógica y metodológica de enseñanza, enfocadas en la valoración intercultural, el cuidado y la conservación del medioambiente, para así contribuir con el sello de la escuela. Se propuso una investigación-acción participativa para aprovechar el amplio espacio de huerto que tiene la escuela y concentrar en su cultivo el foco de trabajo para el diseño didáctico de aprendizajes y de integración curricular de conocimientos escolares.

La propuesta inicial contó con un conjunto de etapas y plan de trabajo que incluía actividades de visita para conocer otras experiencias de huerto escolar, capacitación de docentes, capacitación de alumnos, salidas al terreno para compartir la experiencia, elaboración de material pedagógico y reuniones de coordinación.

Los aspectos favorables en el desarrollo de esta fase fueron la valoración de las iniciativas orientadas a la acción, la adaptabilidad organizativa, el compromiso de docentes, la comunicación entre docentes, el diálogo interinstitucional y la valoración general del proyecto.

- "Es una realidad que en los colegios los tiempos son muy acotados. Teniendo en cuenta esto es que las experiencias de interacción serán de baja calidad, sin embargo una experiencia es una experiencia" (reporte de profesor de la universidad identificado como C. D.).

- "La organización de las clases en que se estaría ausente se solucionó a tiempo dejando las planificaciones y actividades preparadas" (reporte de profesora L. G.).

- "La principal fortaleza es el equipo local. Un equipo comprometido y proactivo, capaz de sacar adelante una iniciativa sin importar los factores adversos" (reporte de profesor C. D.).

- "El equipo de la escuela rural goza de una buena comunicación, por lo tanto, la exposición de las posturas se da de forma natural y ha sido relativamente rápido llegar a acuerdos" (reporte de profesor C. D.).

- "La asesoría de la universidad en primera instancia fue muy pertinente, entregaron el impulso necesario para llevar a cabo la investigación" (reporte de profesor M. R.).

- "Las reuniones de trabajo inicial posibilitaron la reflexión del equipo respecto 
a las características de nuestros estudiantes y las condiciones que ofrece la escuela" (reporte de profesor M. R.).

No obstante, también se observaron aspectos desfavorables. Estos tuvieron que ver con descoordinaciones, falta de recursos y la vulnerabilidad social en que vivían los alumnos como elemento que podría afectar su participación en el proyecto.

- "Por falta de tiempo, dado que los docentes trabajan en aula, no se pudo preparar de manera adecuada a los niños y se decide seleccionar un grupo de cada curso, los que solo en tiempos reducidos se reunieron para dar algunas indicaciones" (reporte de profesora L. G.).

- "En los gastos operacionales de este proyecto no se consideraron las suficientes horas de coordinación" (reporte de profesor C. D.).

- "La escuela rural es una comunidad educativa rural de alta vulnerabilidad, con realidades familiares disfuncionales, condición socioeconómica muy baja" (Acta Rn. de la universidad).

En la fase de convocatoria e inicio del proyecto, el trabajo interinstitucional se focalizó en el huerto escolar como oportunidad para movilizar y dar valor al conocimiento local, la integración interdisciplinar y el trabajo cooperativo. La evaluación retrospectiva reporta dificultades de coordinación, falta de recursos y la vulnerabilidad social de alumnos como barreras que afectan su autoestima y expresividad.

Así, el trabajo cooperativo interinstitucional, a través del proyecto que auspicia la universidad, generó expectativas compartidas entre los participantes, pero también confusión sobre cómo establecer una comunicación eficiente y la intervención de los docentes tanto en el ámbito interno como con la universidad para realizar las tareas propuestas.

Fase de desarrollo: más tareas... más solos En esta fase se llevaron a cabo las actividades previamente planificadas y acordadas entre docentes e investigadores de la universidad. Los docentes realizaron visitas a un huerto escolar con una mayor experiencia y también organizaron a los alumnos de la escuela para que visiten y presenten el huerto escolar de la escuela rural a otra escuela de la comuna. Esta segunda experiencia fue la más comentada y valorada por los docentes en esta fase; apreciaron la experiencia vivida por los niños a través de sus testimonios y la integración de saberes disciplinares que se movilizaron para la organización de la presentación de los alumnos.

- "La experiencia piloto realizada en otra escuela rural con estudiantes expositores fue positiva, a pesar de que el tiempo fue muy reducido para preparar esta actividad y se presentaron ciertas complicaciones que obligaron a improvisar en ciertos ámbitos. Esta exposición fue diseñada y montada por el equipo de escuela rural y ejecutada por los estudiantes de segundo y sexto año básico de nuestra escuela, quienes hablaron sobre las tareas del huerto escolar y realizaron demostraciones de procedimientos de cultivo y exhibición de herramientas de trabajo (reporte de M. R.).

- "Fuimos a explicarles a los niños de la escuela cómo trabajamos en el huerto 
escolar" (niña G. de grupo focal de estudiantes).

- "Explicamos cómo sembrar diferentes tipos de semillas" (niña F. de grupo focal de estudiantes).

Asimismo, valoraron su experiencia como docentes, y la que tuvieron los estudiantes participantes en la demostración de conocimientos de plantas nativas que no suelen ser visibles en el currículum oficial.

- "El proyecto de investigación asociativa universidad-escuela es un aporte al desarrollo pedagógico, ya que abre las puertas para mostrar la labor docente, sistematizando trabajo que se realiza diariamente, que muchas veces pasa desapercibido ante el resto y el conocimiento de la experiencia muchas veces se pierde y no se mejora ni se traspasa" (relato de L. G.).

- "Los resultados obtenidos son bastante positivos, principalmente de parte del aprendizaje y desarrollo de los estudiantes que participaron en la actividad expositiva en otra escuela rural" (reporte de M. R.).

Grupo focal con estudiantes de segundo y sexto básico sobre la experiencia de ir a presentar el huerto escolar a niños de otra escuela:

- "Yo me acuerdo al empezar tenía miedo y después estaba más tranquila" (niña A.).

- "Yo me sentía con nervios, con miedo porque era como la primera vez que vivía como una experiencia así” (niña F.).

- "Igual el huerto es como una manera de cuidar la naturaleza” (niña G.).
- "Y que nosotros mismos podemos aprender lo que no aprendimos en nuestras casas" (niña F.).

Los docentes indicaron que una fortaleza para el desarrollo de la propuesta fue el uso de redes sociales, las que favorecieron el desarrollo de actividades.

- “Se buscó contactos entre las redes cercanas o canales de comunicación con otra escuela rural, por medio de un integrante del equipo, que trabajaba en este establecimiento, además de solucionar la movilización, para el traslado de los niños que iban a exponer" (reporte de L.G.).

Pero también se consolidaron las dificultades. El aspecto desfavorable más señalado por los docentes fue la poca presencia de investigadores de la universidad en el proceso.

- "Hubo un periodo de tiempo en que no contamos con el apoyo de la universidad, las reuniones de coordinación solo se realizaron con el equipo de Escuela Rural, lo que provocó muchas dudas y confusiones para continuar con el proyecto" (reporte de L. G.).

- "La organización del proyecto se vio interrumpida por largos plazos de tiempo en que no hubo comunicación con agentes de la universidad" (reporte de M. R.).

- "Este proyecto fue desarrollado de forma inconsistente. A mitad de proceso, se produjo un vacío temporal en el que solo se avanzó mediante las reuniones mensuales de una hora, por parte del equipo de escuela rural. Este lapso de tiempo sin asesorías debilitó el proceso de ejecución" (reporte de M. R.). 
En la fase de desarrollo, a decir de los docentes de la escuela, el equipo profesional de la universidad no estuvo presente en el trabajo realizado con los alumnos y se echó de menos la asistencia de mayores recursos profesionales: "Es necesario que exista un responsable a cargo del registro audiovisual y la edición del material" (reporte de profesor M.R). En esta fase, los docentes demostraron que estaban orientados a la acción; mientras que la universidad no tuvo la participación esperada por los docentes de la escuela. Se cumplieron las actividades, pero con un desgaste mayor por parte de los docentes y la escuela en general.

Fase de cierre: sistematizar la experiencia y saldar deuda

La fase de cierre refiere al proceso de recoger y sistematizar la experiencia vivida. El reporte fue un compromiso sobre el proyecto, asumido por un investigador de la universidad. Sistematizar la experiencia fue la forma en que la universidad pretendió saldar la deuda de su ausencia en la fase de desarrollo. Así parece que fue tácitamente entendido por todos los participantes, quienes entregaron y construyeron la información para la sistematización.

Entre las fortalezas del proceso total vivido se distinguieron el compromiso del equipo local, el ambiente laboral de la escuela, el entusiasmo de los alumnos y la reafirmación del trabajo de la escuela.

- "El buen ambiente laboral del establecimiento, ya que gracias a eso se logró realizar nuestras reuniones y actividades, siempre hubo un colega dispuesto a quedarse con el curso, a encontrar alguna solución o llegar a un acuerdo" (reporte de profesora L. G.).
- "La disposición, entusiasmo y valor que le dan los niños al huerto escolar, gracias a esto la actividad dio buenos resultados, ya que no contaban con mayor preparación” (reporte de profesora L. G.).

- "Las fortalezas de todo el proceso, en mi opinión, se basan en la reafirmación de un trabajo que viene vislumbrándose y desarrollándose paulatinamente en la escuela rural, en relación al huerto escolar y las múltiples y significativas oportunidades que brinda a los estudiantes para su desarrollo como persona consciente y conocedora del medioambiente" (reporte de profesor M. R.).

Las 10 palabras más asociadas a fortalezas y aspectos favorables de la experiencia fueron el conector para, actividad, proyecto, escuela, estudiantes, trabajo, equipo, huerto, docente, estudiantes, apoyo. Esto se puede visualizar en la nube de palabras elaborada con el programa NVivo 11 Pro (figura 1).

Las palabras más frecuentemente utilizadas para describir las fortalezas del proyecto ponen de relieve las capacidades institucionales y experienciales de la escuela rural, así como la orientación a la acción. El equipo de la universidad no fue mencionado directamente como un actor activo del proceso, pero sí a través de términos funcionales a la acción, tales como apoyo e investigación.

En cuanto a las debilidades del proceso, se manifestó la distancia territorial, que afectó la coordinación interinstitucional; el poco tiempo para hacer innovaciones interdisciplinares, y la ausencia de la universidad en el proceso de desarrollo.

- "Las relaciones laborales tampoco 


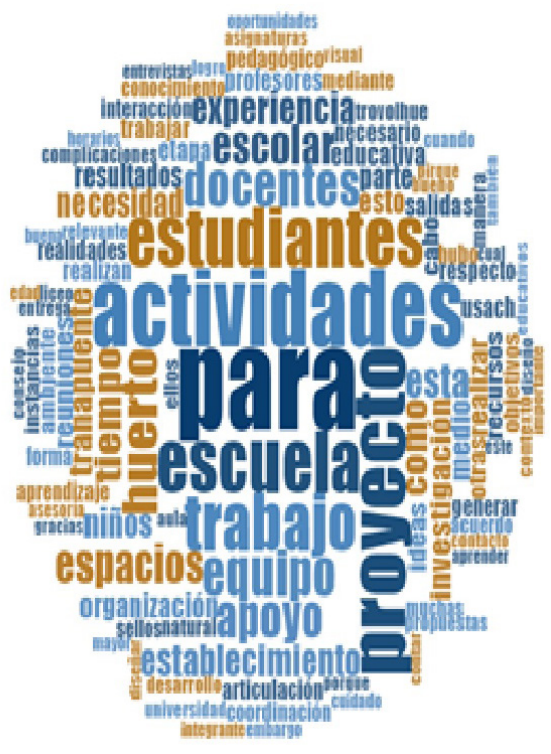

Figura 1. Nube de palabras asociadas a fortalezas del trabajo

resultan a larga distancia. Siempre uno pondrá más empeño que el otro" (reporte de profesor C. D.).

- "El poco tiempo para realizar cualquier actividad dentro del establecimiento, siempre la organización se realiza en recreos, hora de colación y el trabajo pesado se realiza en la casa" (reporte de profesora L. G.).

- "Hubo carencia comunicacional entre el equipo universidad y el equipo escuela rural durante gran parte del proceso. Hizo falta mayor presencia de parte de la universidad. Como equipo de escuela rural, hubo incertidumbres que decayeron la realización del proyecto y no se contó con asesoría en un momento importante" (reporte de profesor M. R.).
Las palabras más utilizadas para dar cuenta de las debilidades del proceso también referían a la acción; pero, además de los 10 términos más frecuentes, emergieron algunos nuevos: equipo, tiempo, universidad (figura 2).

En efecto, la falta de tiempo que tuvieron los docentes para el proyecto y la poca participación de investigadores de la universidad como actor cooperador-activo fueron aspectos que debilitaron el proceso cooperativo.

De la experiencia vivida, también se reconocieron aprendizajes, tales como adaptarse a las circunstancias para desarrollar las actividades; la importancia de planificar dando mayor valor al tiempo para compartir y reflexionar la experiencia; y planear mejor la transferencia de los recursos necesarios para financiar las actividades,

- “La organización de salidas durante la realización del proyecto presentó las

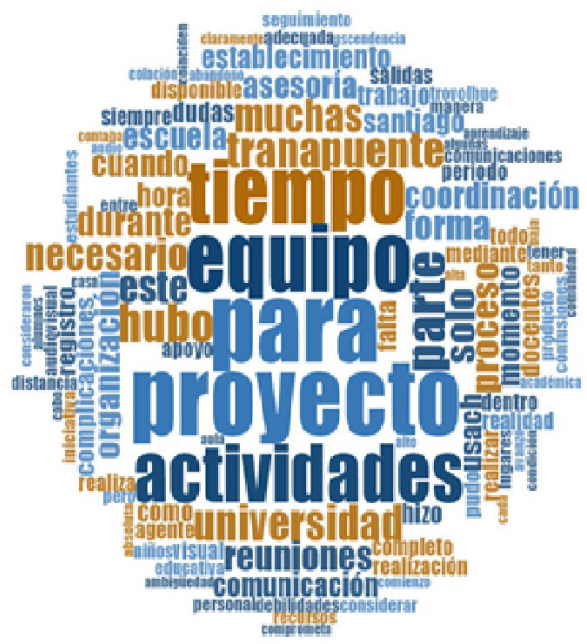

Figura 2. Nube de palabras asociadas a debilidades del trabajo 
complicaciones naturales que ocurren al momento de planificar actividades al interior de un establecimiento educativo: Las fechas propuestas coinciden con otras actividades de la escuela. Los tiempos disponibles de los docentes del equipo son distintos. Esperar la disponibilidad de los lugares de destino. Sin embargo, estas complicaciones fueron resueltas eficazmente, para llevar a cabo las salidas programadas" (reporte de profesor M. R.).

- "Las actividades y visitas realizadas por docentes y de estudiante son enriquecedoras, pero lo son aún más cuando se comparten, por lo que creo necesario destinar un tiempo para compartir lo aprendido con colegas y los estudiantes a sus compañeros. Es relevante también destinar un tiempo específico para la organización y preparación" (reporte de profesora L. G.).

- Los recursos que entrega el proyecto son fundamentales para el desarrollo de las actividades que se realizan, no así los tiempos en que estos se entregan ya que no todos los establecimientos cuentan con 'caja chica' y esto puede retrasar las actividades o provocar la no realización de ellas" (reporte de profesora L. G.).

En el proceso de cierre de la experiencia, la información por sistematizar fue, básicamente, la de los docentes participantes. La investigadora de la universidad presentó ausencias en el proceso y también en el reporte de la experiencia por sistematizar. Esto fue sentido y reconocido por los docentes: "Respecto del cierre del proyecto a mi parecer esta inconcluso, no hubo una instancia de reflexión, quedaron muchas dudas sin respuestas" (reporte de profesora L. G.).

Tiempo para la reflexión es un elemento nuevo que emerge de una revisión de toda la experiencia. La realización de reportes individuales y la formación de grupo focal con los niños son instancias que han podido contribuir a esto, aunque parcialmente.

\section{DISCUSIÓN Y CONCLUSIONES}

El ciclo relatado da cuenta de un proceso de intenciones y realidades del trabajo cooperativo en el que se reportó el logro de objetivos y la ejecución de actividades. Sin embargo, no se cumplieron algunas de las expectativas y compromisos de participación en un proceso conjunto de construcción de innovación educativa culturalmente pertinente. Desde la revisión de estudios previos, se cayó en omisiones de diversos tipos que afectaron el proceso.

Desde esta perspectiva, se presentan tres elementos que sustentan la posibilidad de cooperación universidad-escuela, y cómo han contribuido en esta experiencia para sostener esta acción conjunta.

- El reconocimiento recíproco del valor de la tarea de la propia institución. La experiencia de trabajo en conjunto entre universidad y escuela para metas compartidas es algo necesario y deseable, según reportes de diversos estudios. Esto supone el reconocimiento recíproco de las competencias profesionales para abordar y solucionar problemas. En la experiencia relatada en este reporte, dicho reconocimiento 
refiere a la capacidad de la universidad para asesorar procesos y aportar recursos; y en la escuela, al conocimiento de docentes y estudiantes sobre tipos y variedades de plantas, así como a las posibilidades de organización didáctica de dichos saberes.

El proceso ayudó a que los docentes de la escuela reafirmaran el esfuerzo de dar pertinencia cultural a su trabajo pedagógico, y a desarrollar competencias comunicativas de los saberes de la flora de la región para lograr que conocimientos ancestrales indígenas y vida cotidiana tengan presencia y valor como actividades curriculares.

- El aprecio del otro institucional como colaborador de un proyecto con metas compartidas. El trabajo cooperativo tiene sentido y continuidad cuando el reconocimiento de la diferencia de competencias va de la mano con la valoración de dichas diferencias para alcanzar metas compartidas. La formación de un equipo de trabajo con profesionales de diferentes instituciones -universidad y escuela- es la expresión operativa del otro institucional, con el cual se construye una acción cooperada para alcanzar una meta.

En la presente experiencia es de considerar que dos elementos sostuvieron el aprecio del otro institucional:

- Los recursos humanos. Los profesionales de ambas instituciones y los niños participantes de la escuela.

- Los recursos materiales. El fondo económico que aportó la universi- dad, el amplio espacio de huerto de la escuela.

En el desarrollo del proceso, la universidad fue perdiendo presencia y disminuyendo su aporte de recursos humanos, lo que fue un factor que debilitó al equipo de trabajo en la fase de desarrollo, pues su aporte se vio acotado a la transferencia de los recursos económicos comprometidos.

- El compromiso de los actores educativos interinstitucionales para lograr que los estudiantes alcancen aprendizajes significativos. El término compromiso es recurrente en la literatura de investigación-acción-cooperativa. No es posible la investigación-acción sin compromiso con un ideario educativo. En la experiencia reportada es destacable el compromiso de la escuela y los docentes con la actividad. La universidad y el equipo designado aportaron al impulso inicial. En el discurso de los docentes, los investigadores de la universidad no tuvieron la participación esperada por ellos en el desarrollo de las actividades, y la retomaron la sistematización final. Eso afectó el carácter cooperativo de la investigación y la experiencia posiblemente mutó de una relación cooperativa a una de rendición de cuentas de actividades efectuadas por los docentes en la escuela.

De la experiencia vivida se pueden reconocer dos nudos críticos y extraer aprendizajes con respecto a la implementación de la investigación asociativa universidad-escuela.

- La gestión de contingencias internas de cada institución en la coordinación 
interinstitucional. Universidades y escuelas son instituciones que trabajan con personas $\mathrm{y}$, en consecuencia, tienen dinámicas internas de funcionamiento de mucha contingencia cotidiana. Entre las otras muchas cosas que suceden en las instituciones educativas, la posibilidad de trabajar asociadamente -con todas las ventajas que esto tienesupone niveles de sobrecarga laboral en los participantes. Esto es un elemento que debe considerarse para organizar los tiempos, los espacios y a los profesionales que participan de una investigación asociada. El aprendizaje de esto es conocer los protocolos y tiempos administrativos institucionales que inciden en la colaboración interinstitucional.

- Las diferencias en la semantización de problemas y metas de una intervención. El lenguaje opera en el contexto, $\mathrm{y}$ universidad $\mathrm{y}$ escuela operan en contextos educativos diferentes. En tal sentido, para definir una meta compartida, importa establecer conversaciones recurrentes que conlleven a construir códigos comunes para dar cuenta de la realidad. Lo que para los investigadores de la universidad puede ser un problema educativo no necesariamente lo es para los docentes de la escuela.

El estudio demuestra que los docentes ven los problemas educativos como aquellos relacionados con la acción que resuelve contingencias de afectar el funcionamiento de la escuela para el logro de aprendizajes.
Mientras que, para los investigadores de la universidad, los problemas educativos van en la línea de la comprensión de dichas situaciones. Se puede argüir que acción y comprensión son dos caras de la misma moneda, pero lo que aquí sostenemos es que operan en momentos y espacios diferentes. El aprendizaje es valorar la importancia de clarificar las metas de la colaboración con criterio de realidad por sobre el justo deseo de las grandes transformaciones.

Finalmente, el trabajo cooperativo requiere no olvidar que son dos instituciones - universidad y escuela- que tienen sus propias dinámicas $\mathrm{y}$, en consecuencia, posibilidades y límites de acción. Esto ayuda a evitar la confusión en las metas de la colaboración, que son solo un aspecto de las metas propias de cada institución. Además, conlleva a considerar la importancia de sostener y enriquecer los canales de comunicación y retroalimentación de inicio, desarrollo y resultados de la acción interinstitucional haciendo los cambios necesarios con criterios compartidos.

Se suele decir que el error es un recurso del aprendizaje. No obstante, reconocerlo siempre es doloroso para el aprendiz. El presente reporte da cuenta de lo que sucede cuando esta cooperación no se ajusta a acuerdos compartidos, compromisos asumidos y condiciones institucionales para la investigación-acción que permitan fortalecer la necesaria acción conjunta en el campo educativo. 


\section{REFERENCIAS}

Fals, O. (1999). Orígenes universales y retos actuales de la IAP (Investigación Acción Participativa). Análisis Politico, 38, 71-88.

Francke A, D., y Ojeda, V. P. (2013). Historiografía e historia de mujeres: estrategias para su inclusión en los procesos de enseñanza-aprendizaje en la educación media chilena. Estudios Pedagógicos, 39(1), 361-375. doi:10.4067/S0718-07052013000100021

Gómez, G. R., Flores, J. G., y Jiménez, E. G. (1996). Metodología de la investigación cualitativa. Málaga: Aljibe.

Labrador-Piquer, M. J., y Andreu-Andrés, M. Á. (2014). Investigación-acción para conseguir grupos colaborativos eficaces. Educatio Siglo XXI, 32(3), 75-98.

Martínez, M. C. (2014). Reflexiones en torno a la investigación-acción educativa. CPU-e, Revista de Investigación Educativa, 18, 58-86.

Serrano, G. P. (1994). Investigación cualitativa: retos e interrogantes. I. Métodos. Madrid: La Muralla.

Simón, C., Giné, C., y Echeita, G. (2017). Escuela, familia y comunidad: Construyendo alianzas para promover la inclusión. Revista Latinoamericana de Educación Inclusiva, $10(1), 25-42$. 